\title{
Link Between the Geometrical and the Spectral Transformation Approaches in Scattering Theory
}

Eric Mourre

Centre de Physique Théorique, C.N.R.S., F-13288 Marseille, France

\begin{abstract}
We show how the Enss's geometrical proof of asymptotic completeness may be set on commutator properties.
\end{abstract}

In [1], the author introduces a new method to prove the asymptotic completeness for potential scattering. This method is based on geometrical concepts which are very intuitive and linked to the classical free evolution. Practically the author introduces a decomposition of the identity on $L^{2}\left(\mathbf{R}^{n}\right): \mathbf{1}=P_{\text {out }}+P_{\text {in }}$ such that $\left(\Omega_{\mp}-1\right) P_{\text {in }}$ art are compact and that enables him to prove asymptotic completeness.

We show in this article how to introduce a similar decomposition of the identity $\mathbf{1}=P^{+}+P^{-}$, from the commutator relation $i\left[H_{0}, A\right]=H_{0}$, where $A$ is the well known dilatation group generator on $L^{2}\left(\mathbf{R}^{n}\right)$ (see [2]), and $P^{+}$and $P^{-}$are the spectral projectors of $A$ respectively on the positive and negative parts of the $A$ spectrum.

To prove Lemma 1, we use commutator properties to get interesting differential inequalities, as it has been used in [3] and partially refined in [4], in order to obtain hamiltonian resolvent estimations in the neighbourhood of the continuous spectrum.

\section{Definitions}

Let $\Delta$ be the laplacian on $L^{2}\left(\mathbf{R}^{n}\right), H_{0}=-\Delta$ and $A$ be the generator of the dilatation group on $L^{2}\left(\mathbf{R}^{n}\right)$ normalized in such a way that

$$
e^{+i A \alpha} H_{0} e^{-i A \alpha}=H_{0} e^{-\alpha} \text {. }
$$

Let us note $P^{+}$and $P^{-}$the spectral projectors of $A$ on the positive and negative parts of its spectrum.

Lemma 1. Let $g$ be a regular function with compact support in $\mathbf{R}^{+}-\{0\}$ and $0 \leqq \mu^{\prime}<\mu$.

Then there is a constant $c$ (depending on $g, \mu, \mu^{\prime}$ ) such that

$$
\left\|\chi^{ \pm}(t) \frac{1}{|A+i|^{\mu}} e^{-i H_{0} t} g\left(H_{0}\right) P^{ \pm}\right\| \leqq c \frac{1}{|t|^{\mu^{\prime}}} .
$$


Proof. In the mean value sense on a dense set we get

$$
e^{-i H_{0} t} g\left(H_{0}\right)=\frac{1}{2 \pi i} \int_{-\infty}^{+\infty} d E e^{-i E t}\left(\frac{1}{H_{0}-E-i 0}-\frac{1}{H_{0}-E+i 0}\right) g\left(H_{0}\right)
$$

and

$$
\chi^{ \pm}(t) e^{-i H_{0} t} g\left(H_{0}\right)= \pm \frac{1}{2 \pi i} \int_{-\infty}^{+\infty} d E e^{-i E t} \frac{1}{H_{0}-E \mp i 0} g\left(H_{0}\right) .
$$

If $t>0$ (for instance) we shall write for the moment formally

$$
\frac{1}{|A+i|^{m}} e^{-i H_{0} t} g\left(H_{0}\right) P^{+}=\frac{1}{2 \pi i} \frac{m^{\prime} !}{(i t)^{m^{\prime}}} \int_{-\infty}^{+\infty} d E e^{-i E t} \frac{1}{|A+i|^{m}} \frac{1}{\left(H_{0}-E-i 0\right)^{m^{\prime}+1}} g\left(H_{0}\right) P^{+}
$$

Since $g$ is a function with compact support in $\mathbf{R}^{+}-\{0\}$ an interval $(a, b)$ can be found such that

$$
\int_{\mathbf{R}^{+}-[a, b]} d E\left\|\frac{1}{\left(H_{0}-E-i 0\right)^{m^{\prime}+1}} g\left(H_{0}\right)\right\| \leqq c_{0}
$$

and (1) will be verified for any couple $\left(m, m^{\prime}\right)$ such that

$$
\frac{1}{|A+i|^{m}} \frac{1}{\left(H_{0}-E-i 0\right)^{m^{\prime}+1}} g\left(H_{0}\right) P^{+}
$$

is defined as an operator on $L^{2}\left(\mathbf{R}^{n}\right)$ uniformly bounded in $E \in[a, b]$.

Thus the problem can be reduced to the study of

$$
\frac{1}{|A+i|^{m}} \frac{1}{\left(H_{0}-E-i 0\right)^{m^{\prime}+1}} P^{+}
$$

because $\frac{1}{|A+i|^{m}} g\left(H_{0}\right) A^{m}$ is clearly a bounded operator since $g$ is sufficiently differentiable and

$$
i\left[A, g\left(H_{0}\right)\right]=\left.\frac{d}{d \alpha} e^{+i A \alpha} g\left(H_{0}\right) e^{-i A \alpha}\right|_{\alpha=0}=-H_{0} g^{\prime}\left(H_{0}\right) .
$$

Using analyticity, this property can be extended to real values of $m$. If $m \in \mathbf{R}$ and $n \in \mathbf{N}, m>n$ we are going to prove that $\frac{1}{|A+i|^{m}} \frac{1}{\left(H_{0}-E-i 0\right)^{n}} P^{+}$is
well defined as a bounded operator.

There is a dense set $D$ such that, $\forall \Psi$ and $\Phi \in D$

$$
\begin{aligned}
(\Psi & \left.\mid \frac{1}{|A+i|^{m}} \frac{1}{\left(H_{0}-E-i 0\right)^{n}} P^{+} \Phi\right) \\
\quad & =\lim _{\theta \rightarrow 0^{+}}\left(\Psi \mid \frac{1}{|A+i|^{m}} \frac{1}{\left(H_{0} e^{-i \theta}-E\right)^{n}} e^{-\theta A} P^{+} \Phi\right) ; \quad E>0 .
\end{aligned}
$$

Let

$$
F(\theta)=\frac{1}{|A+i|^{m}} \frac{1}{\left(H_{0} e^{-i \theta}-E\right)^{n}} e^{-\theta A} P^{+} .
$$

$F(\theta)$ is the restriction to the positive pure imaginary axis of the analytic 
function of $z=\theta_{0}+i \theta$ :

$$
\begin{aligned}
F\left(\theta_{0}+i \theta\right) & =\frac{1}{|A+i|^{m}} \frac{1}{\left(H_{0} e^{-\left(\theta_{0}+i \theta\right)}-E\right)^{n}} e^{+i A\left(\theta_{0}+i \theta\right)} P^{+} \\
& =\frac{e^{+i A \theta_{0}}}{|A+i|^{m}} \frac{1}{\left(H_{0} e^{-i \theta}-E\right)^{n}} e^{-A \theta} P^{+} .
\end{aligned}
$$

Then the Cauchy formula implies:

$$
\frac{d}{d \theta} F(\theta)=-\frac{A}{|A+i|^{m}} \frac{1}{\left(H_{0} e^{-i \theta}-E\right)^{n}} e^{-A \theta} P^{+}
$$

and

$$
\left\|\frac{d}{d \theta} F(\theta)\right\| \leqq\left\|\frac{1}{|A+i|^{m-1}} \frac{1}{\left(H_{0} e^{-i \theta}-E\right)^{n}} e^{-A \theta} P^{+}\right\| .
$$

Since $\frac{1}{|A+i|^{z}}$ is analytic for $\left.\operatorname{Re}(z) \in\right] 0, m[$ we can give by interpolation an estimation for (5):

$$
\begin{aligned}
& m-1=0 \frac{1}{m}+\frac{m-1}{m} m ; \text { for } \operatorname{Re}(z)=0 \\
& \left\|\frac{1}{|A+i|^{2}} \frac{1}{\left(H_{0} e^{-i \theta}-E\right)^{n}} e^{-A \theta} P^{+}\right\| \leqq c\left(\frac{1}{E \theta}\right)^{n} ;
\end{aligned}
$$

for $\operatorname{Re}(z)=m$ the norm is precisely $\|F(\theta)\|$; then we get

$$
\left\|\frac{d}{d \theta} F(\theta)\right\| \leqq c(E)\left(\frac{1}{\theta}\right)^{\frac{n}{m}}\|F(\theta)\|^{1-\frac{1}{m}} .
$$

This differential inequality implies that $F(\theta)$ is uniformly Hölder continuous with respect to $\theta \in[0,1]$ for each $m>n$.

Using this estimation in (2) we prove that $\forall m>m^{\prime}+1, t>0$

$$
\left\|\frac{1}{|A+i|^{m}} e^{-i H_{0} t} g\left(H_{0}\right) P^{+}\right\| \leqq c\left(m, m^{\prime}\right)\left(\frac{1}{t}\right)^{m^{\prime}} .
$$

Furthermore, we have $\left\|e^{-i H_{0} t} g\left(H_{0}\right) P^{+}\right\| \leqq\left\|g\left(H_{0}\right)\right\|$; thus, again by a simple interpolation with respect to $\operatorname{Re}(m)$ we prove Lemma 1 .

Lemma 2. If $V(x)=(1+|x|)^{-\mu}\left(f_{p}(x)+f_{\infty}(x)\right.$ where, $\mu>1$ and $f_{p} \in L^{p}\left(\mathbf{R}^{n}\right)$ for some $p>\sup \left\{\frac{n}{2}, 2\right\}, f_{\infty} \in L^{\infty}\left(\mathbf{R}^{n}\right)$ then for any sufficiently regular function $g$ with compact support in $\mathbf{R}^{+}-\{0\}\left(\Omega_{\mp}-\mathbf{1}\right) g\left(H_{0}\right) p^{ \pm}$are compact operators on $L^{2}\left(\mathbf{R}^{n}\right)$.

Proof. $\left(\Omega_{-}-\mathbf{1}\right) g\left(H_{0}\right) P^{+}=i \int_{0}^{\infty} e^{+i H s} V e^{-i H_{0} s} g\left(H_{0}\right) P^{ \pm} d s$.

For any $s>0, V e^{-i H_{0} s} g\left(H_{0}\right) P^{+}$is a compact operator. Furthermore the integral $\int_{0}^{\infty}\left\|V e^{-i H_{0} s} g\left(H_{0}\right) P^{+}\right\| d s$ is well defined since

$$
\begin{aligned}
\left\|V e^{-i H_{0} s} g\left(H_{0}\right) P^{+}\right\| & \leqq\left\|V \frac{1}{\left(H_{0}+1\right)^{\alpha}} e^{-i H_{0} s} g\left(H_{0}\right)\left(H_{0}+1\right)^{\alpha} P^{+}\right\| \\
& \leqq\left\|V \frac{1}{\left(H_{0}+1\right)^{\alpha}}|A+i|^{\mu}\right\|\left\|\frac{1}{|A+i|^{\mu}} e^{-i H_{0} s} g\left(H_{0}\right)\left(H_{0}+1\right)^{\alpha} p^{+}\right\| .
\end{aligned}
$$


From Lemma 1 it is sufficient to verify that $\alpha>0$ can be found such that $V\left(H_{0}+1\right)^{-\alpha}|A+i|^{\mu}$ is bounded on $L^{2}\left(\mathbf{R}^{n}\right)$. This is true for $\alpha=2$ because of the hypothesis made on $V$.

\section{Asymptotic Completeness}

Now we will use the Enss argument [1].

Suppose found $\Psi \in \mathscr{H}_{c}(H)$ such that $(\Psi \mid \Phi)=0 \forall \Phi \in \operatorname{Rang}\left(\Omega_{-}\right)$. Then there is a regular function $g$ with compact support in $\mathbf{R}^{+}-\{0\}$ such that $g(H) \Psi \neq 0$ and $\left(e^{-i H t} g(H) \Psi \mid \Phi\right)=0 \forall \Phi \in \operatorname{Rang}\left(\Omega_{-}\right)$. Using the compactness properties of $g(H)-g\left(H_{0}\right)$, we can find a sequence $\tau_{n} \rightarrow+\infty$ such that:

i) $\left\|\left(g(H)-g\left(H_{0}\right)\right) e^{-i H \tau_{n}} \Psi\right\| \rightarrow 0$;

ii) $\left\|\left(\Omega_{-}-1\right) g\left(H_{0}\right) P^{+} e^{-i H \tau_{n}} \Psi\right\| \rightarrow 0$;

iii) $\left\|\left(\Omega_{+}-1\right) g\left(H_{0}\right) P^{-} e^{-i H \tau_{n}} \Psi\right\| \rightarrow 0$.

Finally we get

$$
\begin{aligned}
0 \neq & \lim _{n \rightarrow \infty}\left\|g(H) e^{-i H \tau_{n}} \Psi\right\|^{2} \\
= & \lim _{n \rightarrow \infty}\left(g(H) e^{-i H \tau_{n}} \Psi \mid \Omega_{-} g\left(H_{0}\right) P^{+} e^{-i H \tau_{n}} \Psi\right) \\
& +\lim _{n \rightarrow \infty}\left(g(H) e^{-i H \tau_{n}} \Psi \mid \Omega_{+} g\left(H_{0}\right) P^{-} e^{-i H \tau_{n}} \Psi\right) .
\end{aligned}
$$

The first right side term is equal to zero by hypothesis. The second one is the limit of the following term:

$$
\left(\Omega_{+}^{*} g(H) \Psi \mid e^{+i H_{0} \tau_{n}} g\left(H_{0}\right) P^{-} e^{-i H \tau_{n}} \Psi\right)
$$

which goes to zero when $\tau_{n} \rightarrow+\infty$ because $\Omega_{+}^{*} g(H) \Psi$ can be approached in norm sense by vectors belonging to the range of $\frac{1}{|A+i|^{\mu}}$, so we get a contradiction.

\section{References}

1. Enss, V.: Asymptotic completeness for quantum mechanical potential scattering. Commun. Math. Phys. 61, 285-291 (1978)

2. Aguilar, J., Combes, J.M. : Commun. Math. Phys. 22, 269-279 (1971)

Balslev, E., Combes, J.M. : Commun. Math. Phys. 22, 280-294 (1971)

3. Mourre, E.: Famille de transformations spectrales. Preprint, Marseille (1976) (unpublished) Mourre, E.: Principe d'absorption limité dans le problème à trois corps en mécanique quantique. Technical reports, Marseille (1978)

4. Mourre, E.: Absence de spectre continu singulier pour certains opérateurs self-adjoints. Preprint, Marseille (1979) 